\title{
Protective Effects of Extracts from Polygonum Hydropiper L on Ethanol-Induced Acute Gastric Mucosal Lesions
} SZ Ren ${ }^{1}$, CL Yang ${ }^{2}, \mathrm{~J} \mathrm{Chen}^{3}, \mathrm{~N}$ Wang ${ }^{1}$, WQ Su${ }^{1}, \mathrm{~L} \mathrm{Sheng}^{1}, \mathrm{ZJ} \mathrm{Ma}^{4}, \mathrm{JH} \mathrm{Lei}^{5}$

\begin{abstract}
Introduction: Free radicals and oxidative stress play a central role in the generation and development of acute gastric mucosal lesions (AGML). Traditional Chinese medicine has its unique effect in removing oxygen free radicals and reducing gastric mucosa injury. Polygonum hydropiper $\mathrm{L}$ is extensively used as a traditional Chinese herbal medicine for treating gastrointestinal diseases in China, the reports shows that it possess various pharmacological activities, especially antioxidant and anti-inflammatory properties.
\end{abstract}

Objective: This study was to investigate the protective effect of extracts from Polygonum hydropiper on experimentally-induced AGML in mice.

Methods: The AGML mice model was induced by ethanol, Glutathione peroxidase (GSH-Px), malondialdehyde (MDA) and superoxide dismutase (SOD) were used to measure the antioxidant activity. The nitric monoxide (NO) and $\mathrm{PGE}_{2}$ contents were tested.

Results: The findings demonstrated that the extracts enhanced the activities of antioxidative enzymes SOD and GSH-Px and decreased the level of MDA in the serum and gastric tissues. The higher-concentration extract $\left(1.78 \mathrm{~g} \mathrm{~kg}^{-1}\right)$ had higher antioxidative activities than the lower-concentration extract $\left(0.89 \mathrm{~g} \mathrm{~kg}^{-1}\right)$. Both extracts increased the production of PGE2and decreased the NO level in gastric tissues. In addition, oral administration of absolute ethanol to mice significantly produced extensive hemorrhagic lesions of gastric mucosa, whereas animals pretreated with extract or ranitidine significantly reduced the formation of gastric lesions compared to control group. The extract could decrease distinctly the acute gastric mucosal damage index compared to the model group. The high-dose extract treatment obviously reduced injuries in the gland region after administration of ethanol compared to the model group.

Conclusion: The results obtained from this study showed that extracts from Polygonum hydropiper L exhibited significant protective effects against ethanol-induced AGML, and these are closely associated with antioxidant effect.

Keywords: Oxidative stress; Polygonum hydropiper L extracts; acute gastric mucosal injury; antioxidation

From: ${ }^{1}$ School of Pharmacy, Hainan Medical University, ${ }^{2}$ College of Animal Sciences, Key Laboratory of Molecular Animal Nutrition, Zhejiang University, ${ }^{3}$ Department of pharmacy, Hainan Provincial People's Hospital, ${ }^{4}$ Department of Human Anatomy, Hainan Medical University, and ${ }^{5}$ Oncology Department, Affiliated Hospital of Hainan Medical University, Haikou, China.

Correspondence: Professor Z Ma, Department of Human Anatomy, Hainan Medical University

Haikou, China. Fax: 86-898-31350722, e-mail: rsz1029@126.com 


\section{INTRODUCTION}

Acute gastric mucosal lesions (AGML) are acute gastric mucosal lesions caused by many kinds of harmful stress factors, and are mainly characterized by gastric mucosal congestion, edema, inflammatory cell infiltration or erosion and superficial ulceration. AGML is one of the most common serious complications in clinical practice, especially for patients with severe burn wounds, major surgery, severe brain injury, severe infection and other urgent and critical populations (1).

The factors that affect the occurrence and development of AGML are extremely complex, but excessive and persistent injury stress is the most important and direct factor to induce the occurrence and development of AGML. During oxidative stress, when the body is subjected to a variety of noxious stimuli, highly reactive molecules in the body, such as reactive oxygen species (ROS) and reactive nitrogen radicals (RNs), are overproduced, beyond the body's ability to eliminate the ROS; this results in an imbalance between the oxidation and antioxidation systems, which can result in intracellular lipid, protein and nucleic acid oxidative damage, eventually leading to apoptosis and tissue and organ damage (2-3). The oxidative stress is induced by injury, infection, ischemia, hypoxia and other factors. In the stress state, a large number of reactive oxygen species (ROS) is generated in the gastric mucosa, and excessive ROS can lead to oxidative stress, including lipid peroxidation of gastric mucosal vascular endothelial cells and gastric mucosa epithelial cells, protein oxidation and DNA damage. Moreover, ROS cause damage to gastric mucosal cells, blood vessels and other structures, which leads to AGML, by acting as a second messenger to activate different redox-sensitive signal transduction pathways, thus destroying the balance of gastric mucosal integrity (4).

The main antioxidant defense enzymes are superoxide dismutase (SOD), glutathione peroxidase (GSH-Px), glutathione reductase and catalase (CAT). Studies show that traditional 
chinese medicine suppresses oxidative stress states and tissue damage by improving the antioxidant enzyme activity to eliminate free radicals, thus inhibiting lipid peroxidation (5). MDA is an important index to reflect the degree of lipid peroxidation in the body.

Polygonum hydropiper L is Polygonum dry grass that is found distributed in the slope and wetland regions and is widely used as a traditional Chinese herbal medicine in China. It has the function of strengthening the spleen and eliminating dampness, and dissipating blood stasis to relieve pain and swelling by detoxification; it is used clinically to treat acute gastroenteritis, diarrhea, dysentery, rheumatism and joint pain (6). Moreover, the chemical composition of Polygonum hydropiper L mainly comprises flavonoids, volatile oil, tannins and other components (7). Studies have shown that flavonoids are the main active substances of Polygonum hydropiper L, which is believed to possess various pharmacological activities such as antioxidant, antiinflammatory, antibacterial and others (8).

Concerning the involvement of oxidative stress in gastric damage, the search for antioxidant substances is valuable, and many reports are available. Despite the evidence for the use of antioxidants, to the best of our knowledge (data retrieval), until now, there are no reports concerning the protective effect of Polygonum hydropiper L against acute gastric mucosal lesion models. Thus, the objective of the study is to explore the effect of Polygonum hydropiper L against oxidative stress and gastric tissue damage induced by $\mathrm{EtOH}$ administration in mice by evaluating markers of oxidative stress along with $\mathrm{PGE}_{2}$.

\section{MATERIALS AND METHODS}


Ranitidine was obtained from Furen Pharmaceutical Group Co., Ltd. Anhydrous ethanol was obtained from Guangzhou Chemical Reagent Factory. Formaldehyde was purchased from Tianjin Damao Chemical Co., Ltd. Superoxide Dismutase (batch No.20160111), glutathione peroxidase (batch No.20160111), malondialdehyde (batch No.20160112), nitric monoxide (batch No.20160112) and Coomassie brilliant blue (batch No.20160107) were purchased from Nanjing Jiangcheng Bioengineering Institute (Nanjing, China).

Kunming mice(18-22g), half male and half female, purchased from Slack JingDa Laboratory Animal Co., Ltd, Changsha, China, [Certificate No: SCXK (Xiang) 2014-0011]. The mice were fed under the condition of controlled temperature $\left(21 \pm 2^{\circ} \mathrm{C}\right)$, relative humidity (approximately 60\% $\pm 10 \%$ ), 12-hour light/dark cycle and automatic ventilation 8-15 times per hour. All animal experiments were approved by the Institutional Animal Care and Use Committee at the Hainan Medical University.

The stem and leaves of Polygonum hydropiper Linn were collected from the Hainan province of China, and its identity was confirmed by our plant taxonomy research group. Voucher specimens were deposited in the School of Pharmaceutical Science, Hainan Medical University.

The air-dried stem and leaves of Polygonum hydropiper L were extracted with water ( $2 \mathrm{~h}$, $98{ }^{\circ} \mathrm{C}$ ) after grinding into powder. The solvent was removed under reduced pressure to afford the water decoction of Polygonum hydropiper L. The same extraction procedures were repeated once, and the obtained decoction was combined and concentrated to a concentration of $2 \mathrm{~g} / \mathrm{ml}$ under reduced pressure, thus obtaining the water extract of Polygonum hydropiper $\mathrm{L}$.

After a 1-week adaptation period, the animals were randomly divided into five groups ( $\mathrm{n}=10$ per group): the control group, model group, ranitidine group, high-concentration water extract $\left(0.89 \mathrm{~g} \mathrm{~kg}^{-1}\right)$ group and low-concentration water extract $\left(1.78 \mathrm{~g} \mathrm{~kg}^{-1}\right)$ group. The 
experimental mice were intragastrically treated with extract $\left(0.89 \mathrm{~g} \mathrm{~kg}^{-1}, 1.78 \mathrm{~g} \mathrm{~kg}^{-1}\right)$ and ranitidine (50 mg kg-1) for five consecutive days before sacrifice, where as those in the control group and model group were provided orally with an equivalent volume of distilled water. On the 6th day, 1h after oral drug administration, all groups of mice except the control group were administered an oral dose of ethanol at $0.2 \mathrm{ml}$, where as those in the control group were administered an equivalent volume of distilled water.

One hour after EtOH administration, the mice were euthanized by deep anesthesia with thiopental (60 mg g kg${ }^{-1}$ intraperitoneally). Blood samples were collected from the orbit vein and centrifuged at $4000 \mathrm{~g}$ for $10 \mathrm{~min}$. The supernatant was collected and set aside at $-20{ }^{\circ} \mathrm{C}$ for the evaluation of serum biochemical indexes. Subsequently, the stomachs were removed from the abdominal cavity. The stomach was washed with a saline solution (sodium chloride $[\mathrm{NaCl}] 0.9 \%$ ), and the glandular portion was separated for macroscopic evaluation. Afterward, the gastric tissue was homogenized in nine volumes of $\mathrm{NaCl} 0.9 \%$ using a homogenizer. A portion of the tissue homogenatewascentrifugedat $3000 \mathrm{~g}$ at $4{ }^{\circ} \mathrm{Cfor} 10 \mathrm{~min}$ to yield a supernatant that was used to measure the levels of MDA and the activity of the antioxidant enzymes SOD and GSH-Px. The remaining homogenate was used to measure the level of prostaglandin $\mathrm{E}_{2}\left(\mathrm{PGE}_{2}\right)$, a protective factor of the gastric mucosa, and nitric oxide (NO), an indicator of mucosal blood flow.

The stomachs were opened along the greater curvature and washed with $0.9 \% \mathrm{NaCl}$. A dissecting microscope was used to observe macroscopic lesions in the gastric mucosa in mice for gastric damage in the glandular part. The severity of gastric mucosal lesions was estimated using a lesion index. The length (mm) of each lesion was measured, and the lesion index was expressed as the sum of the length of all lesions as follows: a score of 1 was given for each $1 \mathrm{~mm}$ of length, 
and the score was doubled for widths of more than $1 \mathrm{~mm}$. Normal stomachs received a score of 0 . Pictures of the gastritis were obtained at this stage.

The activity of SOD in serum and tissue and the measurement of GSH-Px were determined using a kit according to manufacturer's instruction. The content of MDA in the serum and tissue was determined using thiobarbituric acid (TBA).

The tissue content of PGE2 was analyzed by enzyme immunoassays using commercially available reagents according to the manufacturer's instructions. The level of NO in tissue was determined by the Griess assay.

MDA and NO levels, and SOD and GSH-Px activity, were normalized to the amount of stomach protein content. The protein quantification was performed using the Coomassie brilliant blue method, in which the maximum absorbance for the solution of Coomassie brilliant blue dye, due to its anion interaction with serum protein- $\mathrm{NH}_{3}$, occurs at $595 \mathrm{~nm}$.

\section{Statistical analyses}

The experimental results were expressed as the mean \pm SD. A one-way analysis of variance (ANOVA) was used for multiple comparisons followed by application of Duncan's test. P values less than 0.05 were considered significant.

\section{RESULTS}

The experimental results revealed a significant effect of EtOH on gastric tissue at the macroscopic level. The comparisons showed that a 1-h exposure to $\mathrm{EtOH}$ was able to cause injury to gastric 
tissue. Extensively visible edema, hemorrhage and necrosis caused by the ethanol was observed in the model group compared to the control group. The gastric mucosa was brown and lacked flexibility. Compared to the model group, the water extract $\left(1.78 \mathrm{~g} \mathrm{~kg}^{-1}\right)$ treatment group (as shown in Fig. 1) had obviously reduced injury in the gland region after administration of ethanol, as demonstrated by a reduction of mucosal edema, hyperemia and erosion. The water extract $(0.89 \mathrm{~g}$ $\mathrm{kg}^{-1}$ ) treatment group (shown in Fig1) also displayed a certain improvement in the body of stomach. The gastric mucosal injury index increased significantly in the model group, and compared to the model group, the water extract $\left(0.89 \mathrm{~g} \mathrm{~kg}^{-1}, 1.78 \mathrm{~g} \mathrm{~kg}^{-1}\right)$ groups had a distinctly decreased acute gastric mucosal damage index compared to the model group $(\mathrm{P}<0.05)$ (Fig 2).

EtOH administration significantly decreased antioxidant activity in the animals in the experimental conditions used in this protocol. Significant antioxidant activity reduction of SOD and GSH-Px in the serum in the model group was observed compared with the normal control group, whereas the level of antioxidant SOD and GSH-Px was correspondingly decreased in gastric tissues in the model group. However, administration of the water extract $\left(0.89 \mathrm{~g} \mathrm{~kg}^{-1}, 1.78 \mathrm{~g} \mathrm{~kg}^{-1}\right)$ to the animals for 5 days after an ethanol challenge remarkably up-regulated the SOD and GSH-Px level in serum and gastric tissues compared with mice exposed to the model group, and the two changes were consistent in the model and water extract groups. The results showed that the water extracts were able to attenuate the decrease in activity of two antioxidant enzymes (Fig 3A-C).

The end product of membrane lipid peroxidation, MDA, was evaluated by measuring its reaction with thiobarbituric acid. The level of MDA was notably elevated following ethanol treatment. The data suggested that treatment with water extract $\left(0.89 \mathrm{~g} \mathrm{~kg}^{-1}, 1.78 \mathrm{~g} \mathrm{~kg}^{-1}\right)$ resulted in a decrease in MDA in the serum and gastric tissues ( $P<0.05$ for serum and $P<0.01$ for tissue) (Fig 4 A-B). 
PGE2 in the gastric tissues was also detected to evaluate the mucosal protection. As shown in Fig. 5A, ANOVA revealed a significant decrease in the PGE2 levels after EtOH exposure ( $p<$ 0.01). This result confirms the ability of EtOH to reducePGE2 in gastric tissue even $1 \mathrm{~h}$ after its administration. Post-treatment with extract at $0.89 \mathrm{~g} \mathrm{~kg}^{-1}$ and $1.78 \mathrm{~g} \mathrm{~kg}^{-1}$ raised the PGE2 content in the rat stomach compared to the model group. Moreover, this effect of the extract on PGE2 was dose-related; a higher dose promoted the gastric mucosa to produce a greater level of PGE2 (Fig $5 \mathrm{~A})$.

Concerning the level of NO in gastric tissues, a 1-h exposure to EtOH could increase the level of NO in the stomach of mice. However, post-treatment with water extract resulted in a decreased NO level compared to the model group. Furthermore, ranitidine also caused a decrease in $\mathrm{NO}$ (Fig 5B).

\section{DISCUSSION}

Acute gastric mucosal lesions that contribute to really high morbidity and mortality rates, is a multifactor gastric mucosal illness affecting $15 \%$ of seriously ill patients worldwide. The disease is recognized as be due to endogenous or exogenous stimulation, including ethanol exposure of the gastric mucosa, oxidative stress and more (9).

Ethanol consumption induced degeneration and necrosis of gastric mucosal epithelial cells, as well as submucosal vascular endothelial damage and hemorrhage (10-11). Moreover, high concentrations of ethanol can directly damage gastric mucosa barrier, make the intragastric hydrogen ion diffuse into the gastric mucosa, further aggravate the damage of gastric mucosa, eventually leading to gastric mucosal erosion and bleeding (12-13). Accordingly, the gastric lesions model induced by ethanol administration is also an appropriate experimental model to 
evaluate the effects of tested drugs on gastric mucosal lesions. It is well known that ethanol consumption can result in acute gastric mucosal lesions, which is characterized by the generation of reactive oxygen species (ROS), epithelial cell degeneration and necrosis, and an imbalance between defensive and aggressive factors (14).

Previous evidence indicated that oxygen free radical production caused by ethanol treatment and $\mathrm{H}_{2} \mathrm{O}_{2}$ was probably the accountable for the gastric damage. Exogenous $\mathrm{H}_{2} \mathrm{O}_{2}$ can diffuse across membranes quickly and destroy the intracellular antioxidant system, leading to a loss of function and causing cell damage that ultimately destroys cellular function. The current study showed that EtOH administration in mice caused macroscopic lesions to gastric tissue in the model groups; this destruction included congestion, hemorrhage, necrosis and edema. The harmful effect of EtOH on gastric tissue, and its ability to cause hemorrhage and edema, has previously been described. The current findings revealed that the extract significantly attenuated gastric mucosal injury by macroscopic observation, and it lowered the gastric mucosal damage index, clearly indicating that the extract can protect the gastric mucosa and reduce the mucosal damage induced by ethanol.

Under the catalysis of $\mathrm{ADH}$, the ethanol would be transformed to acetaldehyde, acetaldehyde would be converted to into oxygen free radicals. Moreover, the ethanol can induce the infiltration of neutrophil into gastric mucosa with the release of radicals. The generation of reactive oxygen species (ROS) caused by alcohol consumption has been referred to as acute gastric mucosal lesions (15-16]. Oxidative stress has been considered a crucial step in EtOH-induced mucosal damage and is one of the features most likely to precede cell death in gastric mucosal cells (17). When there is impairment of the antioxidant system or an overabundance of ROS 
production, an imbalance between antioxidants and oxidants occurs, and oxidative stress occurs (18).

Cells have a variety of antioxidant defenses to counteract the damage caused by excess ROS. The principal defense systems against ROS are the enzymes superoxide dismutase (SOD), glutathione peroxidase (GSH-Px), glutathione reductase, catalase (CAT) and non-enzymatic antioxidant nutrients (19). Previous research shows that EtOH depletes the enzymes SOD, CAT and GSH-Px, and induces MDA, resulting in oxidative stress $(5,12)$. Due to the convincing evidence of the involvement of ROS in EtOH toxicity, it would be worthwhile to find new antioxidant substances to counteract the injuries caused by $\mathrm{EtOH}$; many reports are available describing such new antioxidant substances [20-21]. In normal condition, the generation and removal of free radicals in the body is in a state of dynamic equilibrium. SOD is an important antioxidant enzyme that can scavenge oxygen free radicals produced from metabolism. Previous studies have shown that gastric mucosal injury was largely related to reactive oxygen species (ROS) (22). SOD mainly converts harmful super oxides into hydrogen peroxides, which are then decomposed to harmless water molecules and carbon dioxide molecule that alleviates gastric damage by preventing oxidative damage (23). SOD activity can directly reflect the body's ability to clear ROS and the body's antioxidant system function. The severity of injury to the gastric mucosa was indicated by the activity of SOD (24). Moreover, lipid peroxidation and lipid-derived free radicals like MDA can results in injury of the mitochondria and lysosomes (25-27), accelerating the gastric mucosal injury.

The level of SOD and MDA are an mportant indicator to evaluate the degree of gastric lesions and the validity of the drug. MDA can be assessed by measuring thiobarbituric acid, which is an index of oxidative stress. We observed that EtOH exposure induced a decrease in SOD 
activity along with an increase in MDA in the gastric tissue and serum of mice. SOD inhibition has already been described after a 1-hour EtOH exposure and might have led to the increase of MDA observed in the model group. Thus, we can speculate that the large number of generated free radicals caused by EtOH overwhelmed the antioxidant capacity of the gastric tissue, inducing MDA and tissue damage. The extract was found to significantly enhance SOD activities while decreasing the MDA content in the serum and stomach homogenate.

It is well known that GSH is an important defense factor in gastric mucosal lesion because it not only possesses direct radical scavenging properties but is also essential for the antioxidant activity of some enzymes (28). It has confirmed that increased GSH-PX activity will play a protective role in gastric mucosa. EtOH also affects GSH-Px activity in gastric tissue. Previous reports described the decline of GSH-Px activity after a 1-h EtOH exposure (29-30). In this study, we observed antioxidant effects of the extract because treatment with this extract raised GSH-Px activity in the tissue homogenate. Moreover, the extract was able to prevent changes in GSH-Px activity.

There is strong evidence that among gastric defenders, ethanol (EtOH) exhausts mucosal defensive actions (31-32). Studies have shown that gastric mucosal injury induced by ethanol leads to decreased prostaglandin E2 (PGE2) content. However, PGE2 is one of the major factors of the gastric mucosal defense.PGE2 can inhibit gastric acid secretion, increase gastric mucosal blood supply, promote mucus and bicarbonate secretion, protect against anti-oxygen free radical damage, enhance epithelial repair and regeneration ability, protect the gastric mucosal cells, maintain mucosal cells function, and enhance the gastric mucosal barrier defense capability; by these mechanisms and more, it plays an important role in the protection of the integrity of the gastric mucosa (33). The current findings revealed that the extract could increasePGE2, a protective factor 
of the gastric mucosa. Its protective effect on the gastric mucosa might occur through increasing the content of PEG2 in the gastric tissue of mice in order to reduce the damage to the gastric mucosa.

Nitric monoxide (NO) is a messenger molecule and inflammatory mediator produced by nitric oxide synthase (NOS). NO can expand the gastric mucosal blood vessels, increase tissue blood supply, regulate mucus secretion, and protect the gastric epithelial cell morphology and function, all of which play a role in protecting the gastric mucosa. However, under stress, iNOS (Inducible nitric oxide synthase, after the cells are stimulated to produce and to play the efficacy) will induce excessive NO production, and the conversion of NO to peroxynitrite causes injury to the gastric tissue (34). The current findings revealed that the extract could maintain the gastric NO content and prevent the oxidative damage caused by the excessive increase in NO content, which is consistent with previous reports (35).

Taken together, these findings indicate that the water extract from Polygonum hydropiper $\mathrm{L}$ was effective in protecting the gastric tissue against mucosal lesions caused by EtOH. The water extract might resist oxidative damage caused by EtOH by increasing the activity of SOD and GSHPx to accelerate free radical scavenging. Moreover, it could exert some protective action on gastric tissue by increasing the PGE2 content and regulating the NO level. Thus, we can propose the possibility that water extracts might present a remarkable protective effect on the gastric mucosa by their antioxidant mechanism.

\section{CONCLUSION}


The results obtained from this study showed that extracts from Polygonum hydropiper L exhibited significant protective effects against ethanol-induced AGML, and these are closely associated with antioxidant effect.

To the best of our knowledge, this is the first report on the effect of Polygonum hydropiper $\mathrm{L}$ on acute gastric mucosal injury. Because Polygonum hydropiper $\mathrm{L}$ is extensively used for acute gastroenteritis in clinical practice, it is important to find information about this Chinese herb as a potential candidate for use as a medicine to treat acute gastric mucosal lesions. Thus, further studies are necessary to clarify the chemical composition of Polygonum hydropiper L and the possible molecular mechanisms of action of this Chinese herbal medicine in the remedy of gastric mucosal lesions.

\section{ACKNOWLEDGEMENTS}

We would like to express our sincerest gratitude to the financial support of National Natural Science Foundation of china and initial funding by Hainan Medical University. The authors declare no competing financial interests.

\section{REFERENCES}

1. Ali T, Harty RF. Stress-induced ulcer bleeding in critically ill patients. Gastro Clin N Am 2009; 38: 245-65.

2. Bartz RR, Piantadosi CA. Clinical review: Oxygen as a signaling molecul. Crit Care 2010; 14: $234-42$. 
3. Valavanidis A, Vlachogianni TK, Fiotakis K, Loridas S. Pulmonary Oxidative Stress, Inflammation and Cancer: Respirable Particulate Matter, Fibrous Dusts and Ozone as Major Causes of Lung Carcinogenesis through Reactive Oxygen Species Mechanisms. Inter J Env Res Pub Heal 2013; 10: 3886-7.

4. Shi HX, Ren JL. Oxygen free radical and gastric mucosal injury. World J Gastroentero 2005; 13: $2582-5$.

5. Wen HJ, Li YQ, Cui YY, Han X. Protective effects of resveratrol on stress induced gastric ulcer in rats. Chin J Hosp Pharm 2014; 34: 15-18.

6. Huang HH, Zhen HS. The recent progress in research on Chinese herbal Polygonum hydropiperlinn. Chin J Ethnomed Ethnopharm 2013; 22: 38-40.

7. Zhang GY, Zeng T. Study on chemical constituents of Polygonum hydropiperlinn. Chem Ind Forest Prod 2005; 25: 21-4.

8. Yang XZ, Hao ZY, Zhu YC, Dong Y, Lin HK. The antioxidant activity of different parts of Polygonum hydropiperlinn. Jiangsu J Agr Sci 2014; 42: 284-6.

9. Grossman MJ, Kurata JH, Rotter JI, Meyer JH, Robert A, Richardson CT et al. Peptic ulcer: new therapies, new disease. Ann Intern Med 1981; 95: 609-27.

10. Li CY, Xu HD, Zhao BT, Chang HI, Rhee HI. Gastroprotective effect of cyaniding 3glucoside on ethanonl-induced gastric lesions in rats. Alcohol 2008; 42: 683-7.

11. Mahmod AA, Sidik K, Salmsh I, Suzainur KAR, Philip K. Antiulcerogenic activity of ageratum conyzoides leaf extract against ethanol-induced gastric ulcer in rats as animal model. Int J Mol Med Adv Sci 2005; 1: 402-5. 
12. Kamath BS, Srikanta BM, Dharmesh SM, Sarada R, Ravishankar GA. Ulcer preventive and antioxidative properties of astaxanthin from Haematococcus pluvialis. Eur J Pharmacol 2008; 590: 387-95.

13. Mota CS, Freitas RB, Athayde ML, Boligon AA, Augusti PR, Somacal S et al. Effect of Vernoniacognata on oxidative damage induced by ethanol in rats. Hum Exp Toxicol 2010; 30: $675-84$.

14. Rozza AL, Moraes TdM, Kushima H, Tanimotoa A, Marques MOM, Bauab TM et al. Gastroprotective mechanisms of citrus lemon essential oil and its majority compounds limonene and $\beta$-pinene: involvement of heat-shock protein-70,vasoactive intestinal peptide, glutathione, sulfhydryl compounds, nitric oxide and prostaglandin E2. Chem Biol Interact 2010; 189: 82-9.

15. Hahm KB, Park IS, Kim YS, Kim JH, Cho SW, Lee AIiL et al. Role of rebamipide on induction of heat-shock proteins and protection against reactive oxygen metabolitemediated cell damage in cultured gastric mucosal cells. Free Radical Bio Med 1997; 22: 711-16.

16. Halliwell B, Gutteridge JMC. Free radicals in biology and medicine. 5th ed. Claredon Press: Oxford 1999; 32: 158-67.

17. Hirokawa M, Miura S, Yoshida H, Kurose I, Shigematsu T, Hokari R et al. Oxidative stress and mitochondrial damage precedes gastric mucosal cell death induced by ethanol administration. Alcohol Clin Exp Res 1998; 22: 111S-114S.

18. Amaral GP, Carvalho NR, Barcelos RP, Dobrachinski F, Portella RdL, da Silva MH et al. Protective action of ethanolic extract of Rosmarinus off icinalis L. in gastric ulcer prevention induced by ethanol in rats. Food Chem Toxicol 2013; 55: 48-55. 
19. Fang YZ, Yang S, and Wu G. Free radicals, antioxidants, and nutrition. Nutrition 2002; 18: $872-79$.

20. Al Batran R, Al-Bayaty F, Jamil Al-Obaidi MM, Abdualkader AM, Hadi HA, Ali HM et al. In vivo antioxidant and antiulcer activity of Parkia speciosa ethanolic leaf extract against ethanol-induced gastric ulcer in rats. PLoS One 2013, 8: e64751-e64751.

21. Viana AFSC, Fernandes HB, Silva FV, Oliveiraa IS, Freitasa FFBP, Machadoa FDF et al. Gastroprotective activity of cenostigma macrophyllumTul. var. acuminate Teles Freire leaves on experimental ulcer models. J Ethnopharmacol 2013; 150: 316-23.

22. EI-Maraghy SA, Rizk SM, Shahin NN. Gastroprotective effect of crocin in ethanol-induced gastric injury in rats. Chem Biol Interact 2015; 229: 26-35.

23. Ma L, Liu J. The protective activity of Conyza blinii saponin against acute gastric ulcer induced by ethanol. J Ethnopharmacol 2014; 158: 358-63.

24. Nartey ET, Ofosuhene M, Agbale CM. Anti-ulcerogenic activity of the root bark extract of the African Iaburnum "Cassia sieberiana" and its effect in the antioxidant defence system in rats. BMC Complem Altern M 2012; 12: 247-58.

25. Amr M, Abbas, Hussein, Sakr. Effect of selenium and grape seed extract on indomethacininduced gastric ulcer in rat. J Physiol Biochem 2013; 69: 527-37.

26. Kwiecien S, Brzozowski T, Konturek SJ. Effects of reactive oxygen species action on gastric mucosa in various models of mucosal injury. J Physiol Pharmacol 2002; 53: 39-50.

27. Kanter M, Demir H, Karakaya C, Ozbek H. Gastroprotective activity of nigella sativa L ion and its constituent, thymoquinone against acute alcohol-induced gastric mucosal injury in rats. World J Gastroenterol 2005; 11: 6662-6. 
28. Dickinson DA, Forman HJ. Cellular glutathione and thiols metabolism. Biochem Pharmacol 2002; 64: 1019-26.

29. Lee IC, Baek HS, Kim SH,Moon C, Park SH, Kim SH et al. Effect of diallyl disulfide on acute gastric mucosal damage induced by alcohol in rats. Hum ExpToxicol 2014; 34: 227 239.

30. Moran LK, Gutteridge JM, Quinlan GJ. Thiols in cellular redox signalling and control. Curr Med Chem 2001; 8: 763-72.

31. Bagchi D, Carryl OR, Tran MX, Krohn RL, Bagchi DJ, Garg A et al. Stress, diet and alcohol-induced oxidative gastrointestinal mucosal injury in rats and protection by bismuth subsalicylate. J Appl Toxicol 1998; 18: 3-13.

32. Vucević D, Mladenović D, Ninković M, Stanković M, Jorgacević B, Stanković M et al. Influence of aging on ethanol-induced oxidative stress in digestive tract of rats. Hum Exp Toxicol 2013; 32: 698-705.

33. Qu Y, Cai LP, Zheng HX, Jiang N, Lin SR, Sun YF et al. Effect of XiaoYongKuideKang on expressions of PGE2 and EGF in rat ulcer model. China J Tradit Chin Med Pharm 2011; 26: $26-37$.

34. Hua W. Animal model and pathogenesis of gastric mucosal injury induced by psychological stress. Foreign Med: Digest Dis 2002; 3: 143-5.

35. Zhou QH, Wang HM. Protective effect of Salvia miltiorrhiza on gastric mucosa. Chin J Mod Med 2008; 18: 582-5. 


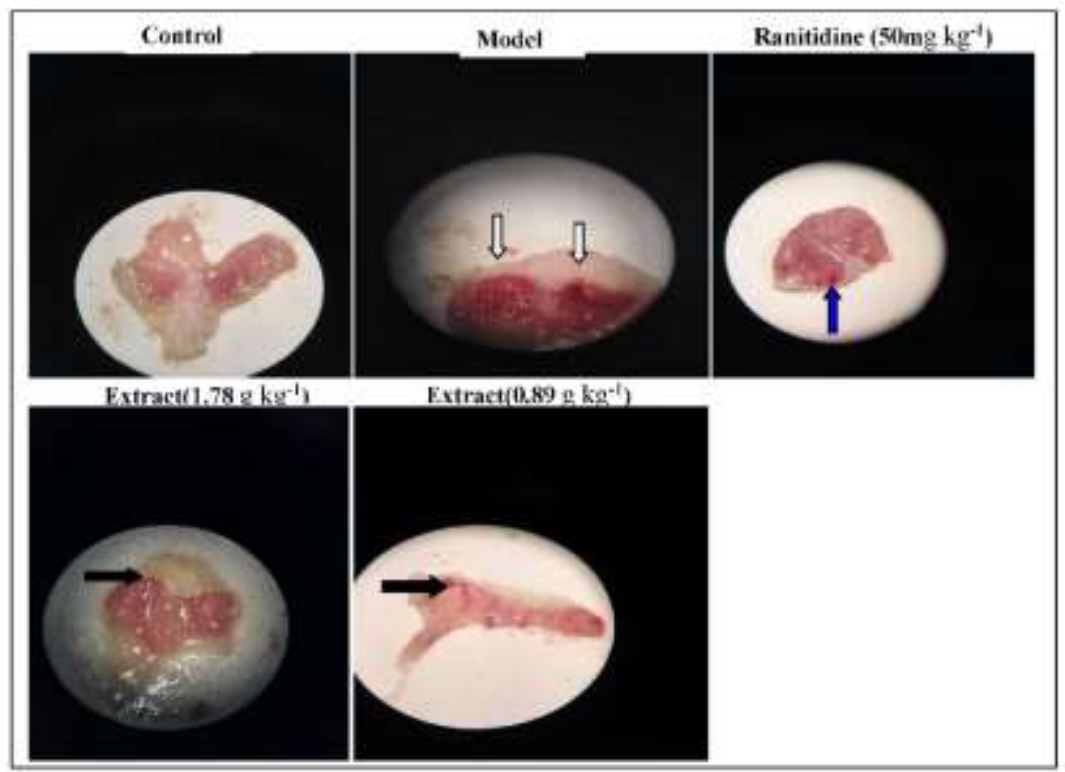

Fig.1: Gross evaluation of gastric mucosal lesions of mice in different groups $(\times 10)$. Effect of extract on ethanol-induced gastric mucosal lesions in mice. The control group shows no visible injuries. The black arrow in the extract $\left(0.89 \mathrm{~g} \mathrm{~kg}_{-}{ }^{1}, 1.78 \mathrm{~g} \mathrm{~kg}_{-}{ }^{1}\right)$ groups and the blue arrow in the ranitidine group indicate injuries in the stomach, whereas the white arrow in the model group indicates injury of the gland region. 


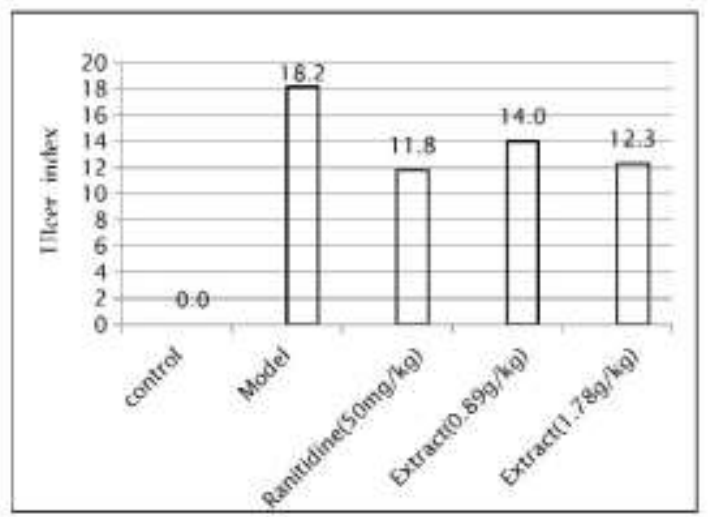

Fig. 2: Score of gastric tissue injury from mice treated with EtOH and/or extract. Data are expressed as the means \pm SDs. \#\# $p<0.01$, significantly different from the values in the control group; and $* * p<0.01$, significantly different from the values in the model group $* p<0.05$, different from the values in the model group. 


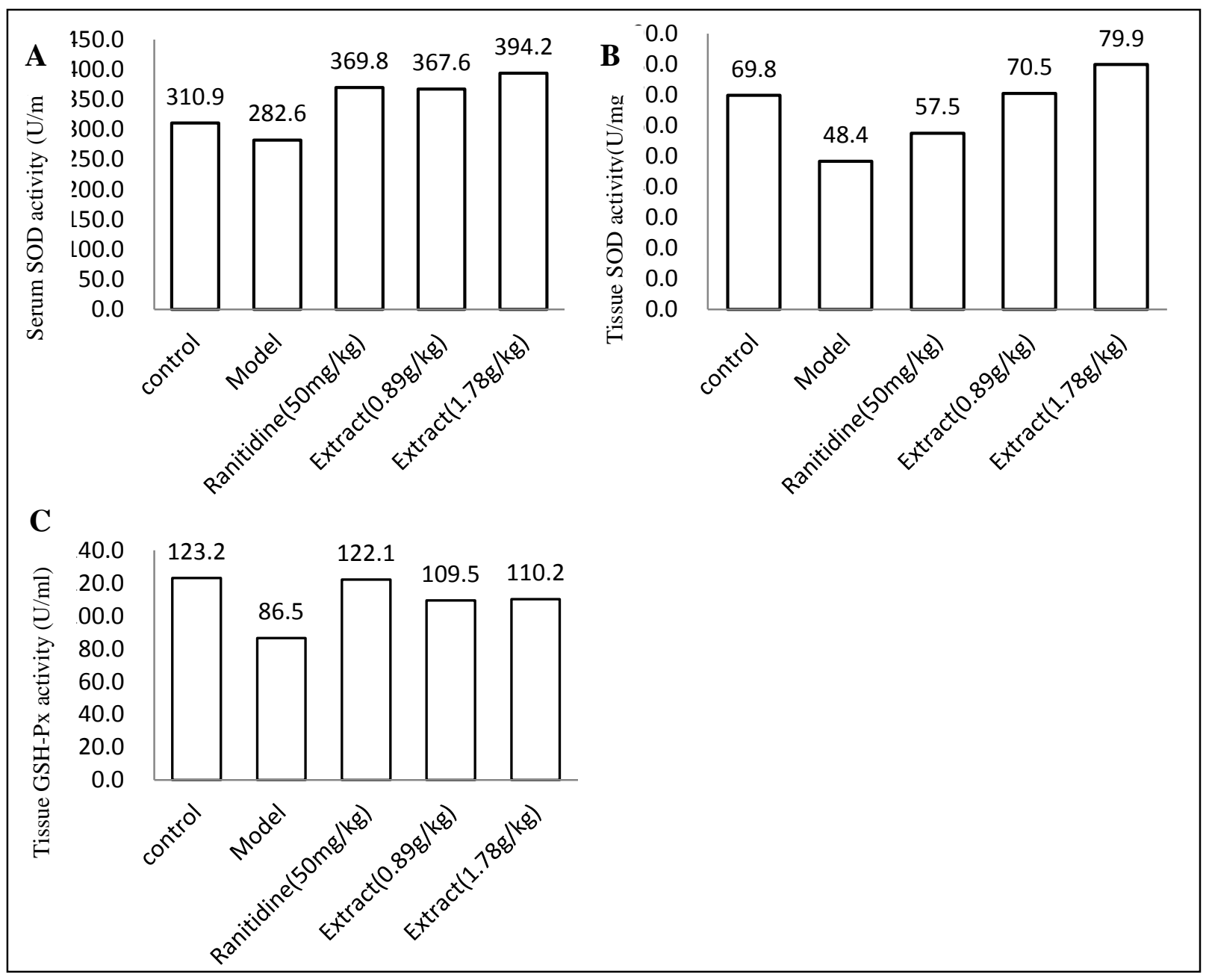

Fig. 3: Measurements of antioxidant activity. Influence of extract treatment on oxidative stress. The improved SOD in serum (A) and tissue (B) and GSH-Px(C) activity in tissue revealed the positive effect of the extract on anti-oxidation. Values represent the means \pm SDs and are representative of two independent experiments. ${ }^{\# \#} p<0.01$, significantly different from the values in the control group; and $*^{*} p<0.01$, significantly different from the values in the model group. $* p<0.05$, different from the values in the model group. 


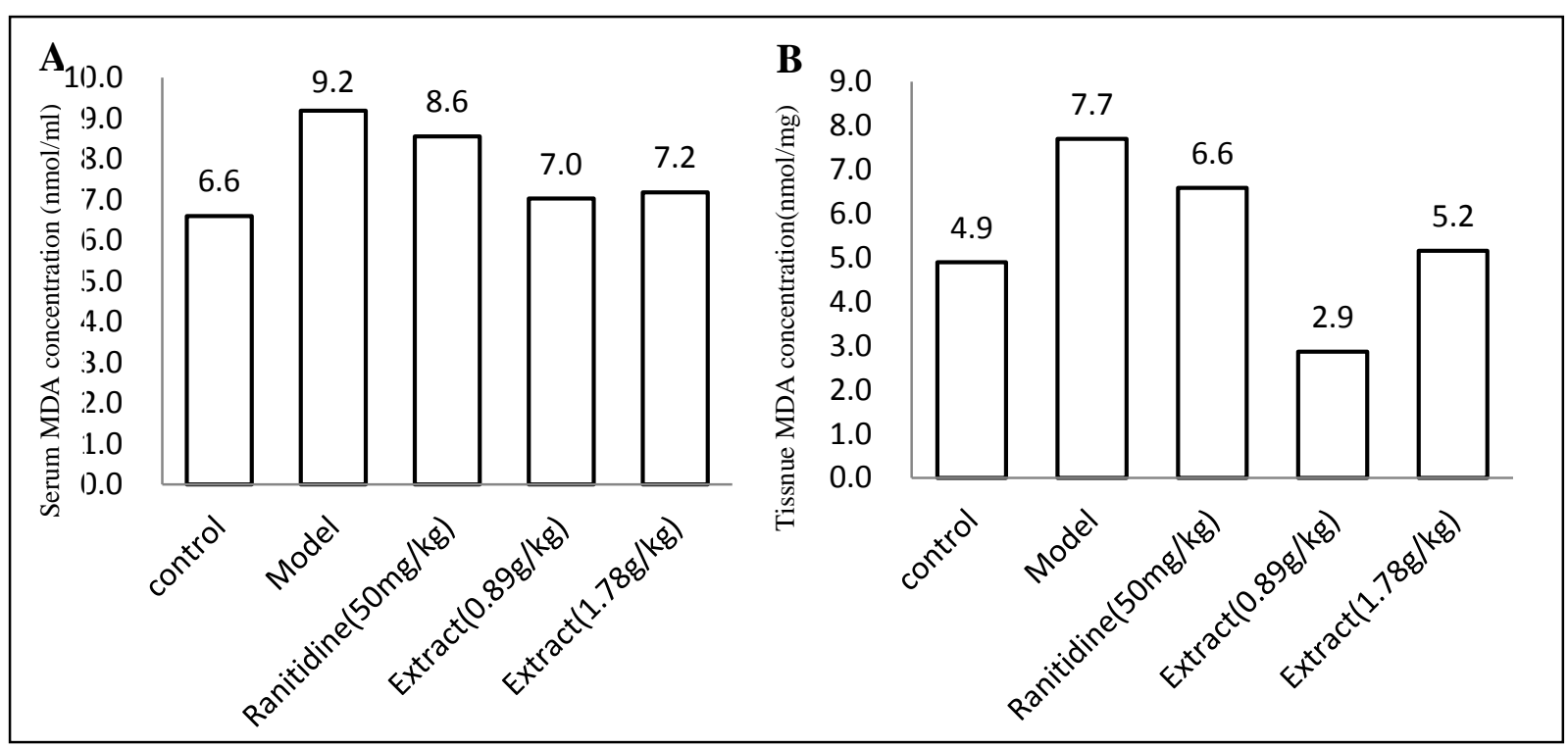

Fig. 4: Measurements of MDA. Influence of extract treatment on MDA content in the serum (A) and tissue (B). The decreased MDA content revealed the positive effect of extract on anti-oxidation. Values represent the means \pm SDs and are representative of two independent experiments. ${ }^{\# \#} p<$ 0.01 , significantly different from the values in the control group; and $* * p<0.01$, significantly different from the values in the model group. ${ }^{*} p<0.05$, different from the values in the model group. 


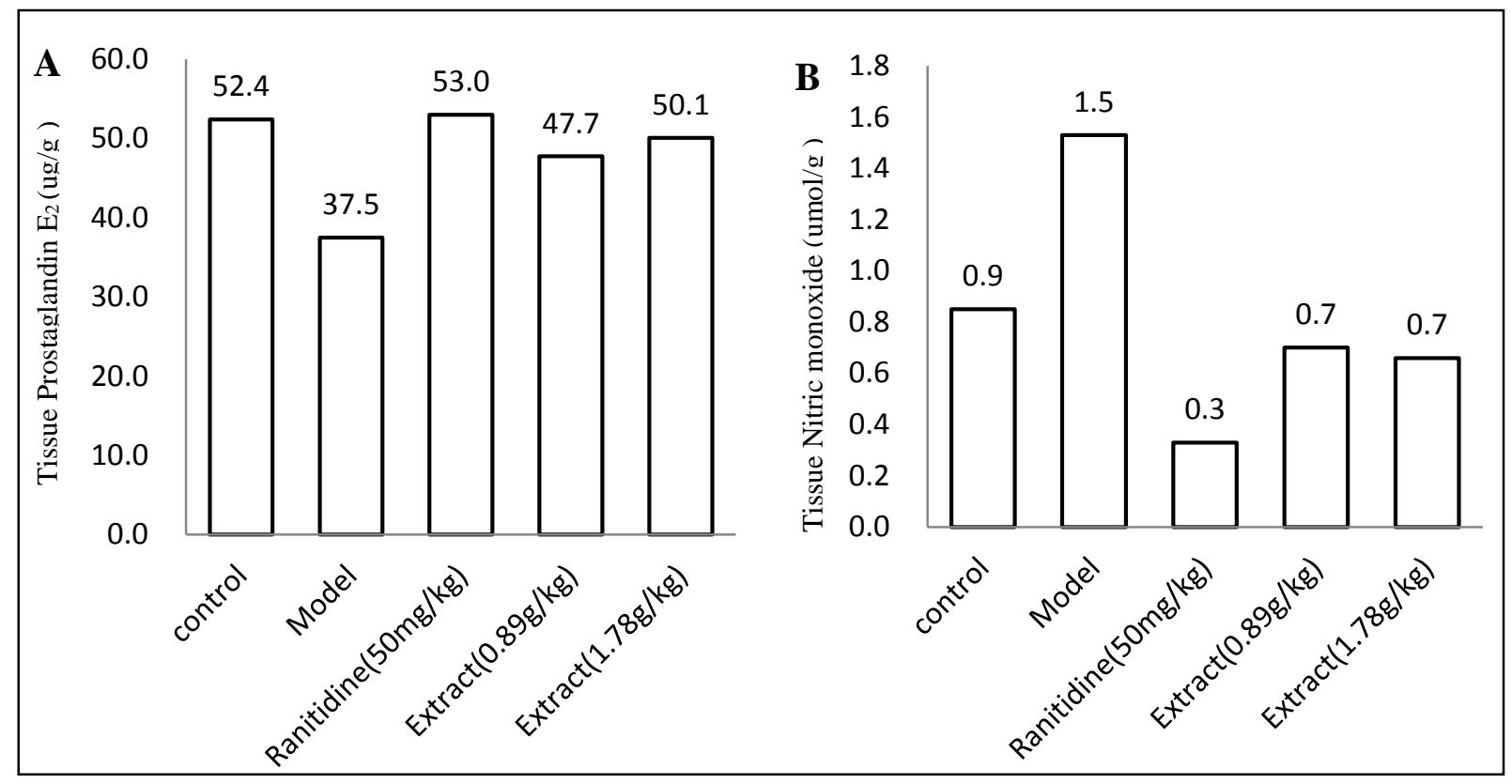

Fig 5: Levels of PGE2 (A) and NO (B) in gastric tissue from mice treated with EtOH and/or extract. Data are the means \pm SDs. ${ }^{\#} p<0.01$, significantly different from the values in the control group; and $* * p<0.01$, significantly different from the values in the model group. $* p<0.05$, different from the values in the model group. PGE2: prostaglandin E2; NO: nitric monoxide. 